\title{
Acidic Alumina Catalysed One Pot Synthesis and Antimicrobial Activity of 1,3,6-Trisubstitutedpyrimidine-2,4-dione Uracils.
}

Anil Kumar ${ }^{1}$,Punam ${ }^{2}$, Sharwan K Dewan ${ }^{3 *}$
${ }^{1}$ Department Of Chemistry, AIJHM PG College, Rohtak, Haryana, Pin-124001
${ }^{2}$ Department of Chemistry, MahaveeraSwami Institute Of Technology, Jagdishpur, Sonepat, Haryana
${ }^{3}$ Department of chemistry, M D University, Rohtak, Haryana, Pin-124001

\section{ABSTRACT}

Acidic aluminacatalyzes the rapid synthesis of 1,3,6-Trisubstituted pyrimidine-2,4-diones by condensation of symmetrically disubstitutedureas with betaketoesters under solvent-free conditions.

\section{Graphical Abstract}<smiles>[R]NC(=[X])N[3H]</smiles>

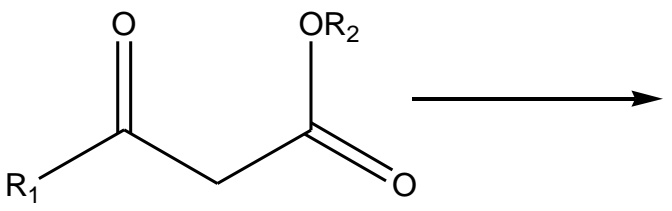<smiles></smiles>

KEYWORDS :1,3,6-Trisubstitutedpyrimidine-2,4-diones, 1,3,6-trisubstituted uracils, methylacetoacetate, ethylbenzoylacatate, solvent-free synthesis, dry media, rapid synthesis, closed Teflon vessel, Acidic alumina.

\section{INTRODUCTION}

Compounds related to the title heterocycles have been found to be associated with attractive pharmacotherapeutic profiles such as analgesic, anti-inflammatory, and anti-pyretic biological profiles.[1-2] The title compounds, 1,3,6-trisubstitutedpyrimidine-2,4-diones, have been synthesized by methods such as by the condensation between the monosubstitutedureas and the diketene, by condensing the monosubstitutedureas and ethylacetoacetate in the presence of conc. $\mathrm{H}_{2} \mathrm{SO}_{4}$.[2-5]These methods yield 1 or 3-substituted-6-methyl uracils which are subsequently alkylated to give the 1,3-disubstituted-6-methyluracil. A recent method for the synthesis of these compounds involves the condensation of a disubstituted urea with an excess of acetic anhydride in presence of 4-methylpyridine solution but the method gives moderate yields and includes a series of tedious extractions work-up.[6] In general, the reported methods suffer from drawbacks like many steps, low 
yields and long reaction times which prompted us to develop new and rapid methods for the synthesis of the title compounds, the 1,3,6-tisubstitutedpyrimidine-2,4-diones.

\section{MATERIALS AND METHODS}

. The NMR spectra were recorded at a $400 \mathrm{MHz}$ Bruker NMR spectrometer . The chemical shifts are reported in ppm and were measured in deuterated chloroform and TMS as an internal standard. TLC was used for monitoring the reaction. The substrates were procured from Aldrich and their purity confirmed by physical and spectroscopic analyses before use. 1,3--Dialkylurea and methylacetoacatate (MAA) or ethylbenzoylacetate (EBA) (1 mmol) and the catalyst (100mg) were taken in a $25 \mathrm{~mL}$ Pyrex beaker in a Teflon bath and the mixture microwaved, with the reaction being monitored by thin Layer Chromatography. The crude product was purified by column chromatography (CCl4/ethylacetate, 94/6) as eluant over silica gel to afford the desired product. The structures of all the products were unambiguously confirmed by spectroscopic and physical data as reported earlier

\section{RESULTS AND DISCUSSION}

We are these days interested in carrying out organic synthesis under solvent- free conditions, and using a catalyst if the reaction so demands and employing the technique of heating by microwaves i.e. under green chemistry conditions rather than under the classical reaction conditions that involves the use of solvents.[7-11] Therefore, we aimed at developing the green rapid methods for the synthesis of the title pyrimidine-2,4-diones and we envisioned their rapid synthesis from a betaketoester like methylacetoacetate, ethylbenzoylacetate and a symmetrically disubstituted urea under dry media conditions.

Recently, we reported a rapid synthesis of 1,3-dialkyl-6-phenylpyrimidine-2,4-diones from the dialkyl urea and the betaketoester in the absence as well as in the presence of the zeolite, silica gel, sodium chloride, Mont-K10, Mont-KSF, catalysts under closed vessel conditions[13]. In this paper, we report the synthesis of the title compounds by the condensation method from a betaketoester and a dissymmetric urea by using acidic alumina, an inexpensive, non-corrosive, non-toxic and environmentally benign substance containing both the desired Lewis and Bronsted acidities which are likely to catalyse the condensation reaction in view of the mechanism involving nucleophilic attack of the urea nitrogen on to the electrophilic carbon of the keto moiety of the betaketoester.

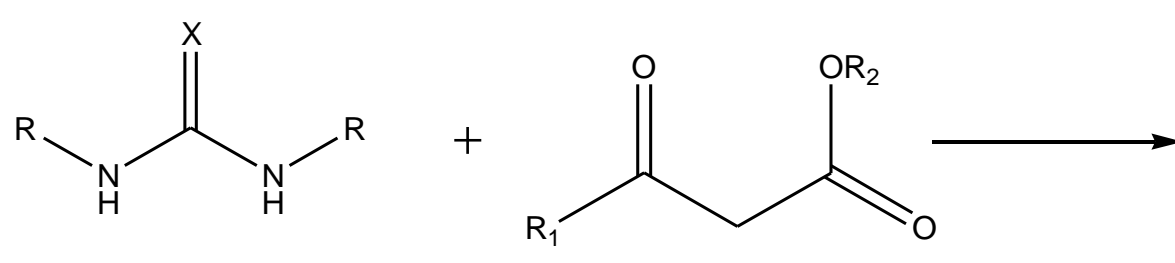

$$
\mathrm{R}=\mathrm{Me}, \text { Et, Allyl, benzyl } \quad \mathrm{X}=\mathrm{O}
$$

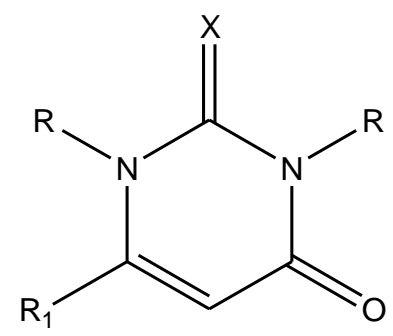

$\mathrm{R}_{1}=\mathrm{Ph}, \mathrm{Me} \quad \mathrm{R}_{2}=\mathrm{Me}, \mathrm{Et}$ 
The investigations were initiated by microwaving a mixture of ethylbenzoyl acetate (EBA) and 1,3dimethylurea (DEU) (taken in 1:1 molar ratio) and MontK-10 (100mg) in an open vessel at various temperatures. Monitoring of the reaction by thin layer chromatography (TLC) showed that the reaction did not occur to any appreciable extent under these conditions. Adjusting the substrate ratio from 1:1 to 1:2 or 1:3 also did not prove successful. However, when the reaction was carried out in a Teflon bath that was fitted with a security disk that could resist pressures up to 10 bars, the desired product, 1,3-dimethyl-6-phenylpyrimidine-2,4dione was formed in $88 \%$ yield after column chromatography compared to $76 \%$ yield without the presence of catalyst. Similarly, the condensation of diethylurea (DEU) with ethylbenzoyl acetate (EBA) gave the 1,3diethyl-6-phenylpyrimidine-2,4-dione in $81 \%$ yield, while the yield of the product in the absence of the catalyst was $72 \%$ only. The 1,3-dibenzyl-6-phenylpyrimidine-2,4-dione from 1,3-dibenzylurea (DBU) and ethylbenzoyl acetate (EBA) was obtained in $91 \%$ isolated yield compared to $80 \%$ in the absence of the catalyst. Encouraged by these results and in order to extend the versatility of the above method and to introduce diversity in the target uracils accessible from the above developed novel one pot method, we decided to attempt the condensation of another readily available beta-ketoester, methylacetoacetate (MAA) with ureas such as DMU, DEU and DAU to obtain the corresponding heterocyclic products. Thus, the condensation of DMU with MAA in the presence of the catalyst gave the 1,3,6-trimethylpyrimidine-2,4-dione in $83 \%$ yield, whereas the yield of the product obtained without the use of the catalyst was only $71 \%$. Similarly, the yield of the condensation product, 1,3-diethyl-6-methylpyrimidine-2,4-dione from DEU and MAA was $76 \%$, while the yield in the absence of the catalyst was only $62 \%$. The condensation of $1,3-$ diallylurea (DAU) and methylacetoacetate (MAA) gave the desired product, 1,3-diallyl-6-methylpyrimidine2,4-dione in $91 \%$ isolated yield, while the yield obtained in the absence of the catalyst was $83 \%$. The yield of the products obtained in the presence and absence of the catalyst are collected in Table 1

Table 1: YIELDS OF THE PRODUCTS IN THE ABSENCE AND PRESENCE OF THE Acidic Alumina

$-$

$\begin{array}{llll}\text { Urea } & \text { Betaketoester } & \text { No Catalyst } & \mathrm{Al}_{2} \mathrm{O}_{3}\end{array}$

$\begin{array}{lccc}\text { DMU } & \text { MAA } & 71 \% & 83 \% \\ \text { DEU } & \text { MAA } & 62 \% & 76 \% \\ \text { DMU } & \text { EBA } & 76 \% & 88 \% \\ \text { DEU } & \text { EB } & 72 \% & 81 \% \\ \text { DBU } & \text { EBA } & 80 \% & 91 \% \\ \text { DAU } & \text { MAA } & 83 \% & 91 \%\end{array}$


As can be seen, the yields of the title heterocyclic products, the 1,3,6-trisubstitutedpyrimidine-2,4-diones were as anticipated better (76-91\%) in the presence of the catalyst than those obtained in the absence (62-83\%) of the catalyst and the time required for completion of the reactions were also observed to be lower. Clearly, acidic alumina has almost shown same catalytic activity as the acidic Mont-K10 (77-92\%) as already reported by us.

\section{CONCLUSIONS}

We have developed a new green rapid one-pot method for the synthesis of 1,3,6-trisubstitutedpyrimidine-2,4diones from the condensation between a 1,3-dialkyl urea and a betaketoester in high yields (70-88\%) in the presence of the acidic alumina catalyst.

\section{REFERENCES}

[1] D. Cole, A.J. Foster, S.W.Freeman, P.E.Murray ,I.J.Stanford, , Anti-Cancer Drug Das, 1999,

14,383;S. Senda, K.J. Hirota, Med Chem, 1972, 15, 471

[2] ] J.A. Patel, N. B. Patel, , J Applicable Chemistry, 2018, 7,45-51.

[3] A Suzui , S Senda , CA 1958, 52, 11972

[4] S Senda , A Suzui, CA 1959, 53, 10237

[5] S Senda , A Suzui, Chem Pharm Bull, 1958,6,479

[6] H Egg, I Volgger, Synthesis, 1982, 1071

[7] S K Dewan, Indian J Chemistry, Section B, 2006, 45, 2337.

[8] S K Dewan, R Singh, A Kumar, Arkivoc, 2006, 2, 41.

[9] S K Dewan, Synth Commun, 2004,34, 2025.

[10] S K Dewan, R Singh, A Kumar, Synth Commun, 2003, 33, 2, 41.

[11] S K Dewan, Punam, A Kumar, J Applicable Chemistry, 2013,2,714

[12] S K Dewan, Punam, J Applicable Chemistry, 2014, 3, 639

[13] Punam, A Kumar, SK Dewan , J Applicable Chemistry, 2014, 3, 639 